\title{
CATÉCHISME POLITIQUE
}

OU

\author{
ÉLÉMENS \\ DU DROIT PUBLIC ET CONSTITUTIONNEL DU \\ CANADA
}

\section{Par A. Gérin-Lajoie}

Réimpression publiée sous les auspices $d u$ Conseil Canadien de Recherche en Sciences Sociales de la Maison des Sciences de l'Homme, Paris $d u$ Conseil Canadien de Recherche sur les Humanités de la Toronto Public Library

S. R. PUBLISHERS LIMITED

JOHNSON REPRINT CORPORATION MOUTON \& CO. N.V. 
Imprimé aux Etats-Unis

S. R. Publishers Ltd.

East Ardsley, Wakefield

Yorkshire, England
Johnson Reprint Corporation

111 Fifth Avenue

New York, N. Y. 10003, U.S.A.

Mouton \& Co. N.V.

Herderstraat 5

The Hague, Netherlands

Réimpression 1967 